\title{
Evaluation of silver nanoparticles caffeic folate complex compound as new potential therapeutic agent against cancer xenograft in mice
}

Tahany S. Abdelwahab, Tahany S. Abdelwahab and Mohga S. Abdallah

Faculty of Science, Helwan University, Helwan, Egypt

\section{III}

Background: In the last few decades the properties of silver compounds have been of interest as potential cancer treatments. Aim: The present work was designed to study the effect of new conjugated caffeic and folic acid with silver nanoparticle with definite molecular size applied with and without gamma radiation exposure, as an antitumor agent against experimentally induced Ehrlich tumor and attempted to identify their potential molecular mechanisms of action throughout determination of anti-tumor activities using MTT cytotoxic assay against two human carcinoma cell lines in vitro. Materials and Methods: Apoptosis analysis by flow cytometry through caspase-8, caspase- 3 and TNF determine in vivo. Adult female albino mice were used and divided into five groups, Animals were scarified and the following parameters were estimated, glutathione, glutathione peroxidase, superoxide dismutase in blood and caspase8, caspase 3 and TNF from scarified tumor tissue. The tumor specimens were processed for histopathological examination. Results: Nano-silver folate caffeic (NSFC) complex compound treatment resulted in a growth inhibition in Hep-G2 and MCF7 cells (IC50 $7.70 \mu \mathrm{M}$ and $14.50 \mu \mathrm{M}$, respectively). Flow cytometric analysis revealed that (NSFC) with radiation exposed has apoptotic effect at caspases 8,3 and TNF more than any compound of them alone. That disturbance was found to be associated with a kinetic induction of apoptosis and showed modulation of antioxidant system beside its high percentage of necrotic cells by histopathological studies. Conclusion: The novel synthetic nano-silver folate Caffeic complex compound may potentially present a new hope for the development of breast cancer therapeutics, which should attract further scientific and pharmaceutical interest.

Keywords: Apoptosis; Conjugated caffeic and folic acid; Radiation; Silver nanoparticles; Tumor 